\title{
Chronic Traumatic Encephalopathy: Connecting Mechanisms to Diagnosis and Treatment
}

\author{
Christina Milani $^{1 *} \&$ Nafisa M. Jadavji ${ }^{1}$
}

Chronic traumatic encephalopathy is a progressive neurodegenerative disease that has been linked to the incidence of repetitive mild traumatic brain injuries. As chronic traumatic encephalopathy has no formal diagnosis or treatment, current research is striving to better understand its neuropathology in order to develop effective diagnostic and treatment strategies. This review will outline recent research findings in the understanding of the neuropathological mechanisms of chronic traumatic encephalopathy, and connect these findings to advancements in the diagnosis and treatment of the disease. With the emergence of more sophisticated technology, neuroimaging techniques have shown promise as prospective diagnostic tools. Functional neuroimaging techniques that allow for the observation of taskrelated brain activity such as functional magnetic resonance imaging (fMRI) and positron emission tomography (PET) imaging have provided significant insight into the progression of chronic traumatic encephalopathy. Additionally, a branch of magnetic resonance imaging (MRI) called diffusion tensor imaging (DTI) is currently being used to assess white matter integrity, which is often compromised in cases of repetitive mild traumatic brain injury and may be indicative of an increased risk for developing chronic traumatic encephalopathy and other neurodegenerative diseases. Several forms of pharmacotherapy, including lithium treatment and monoacylglycerol antagonists, have been suggested to target the common neuropathological markers of chronic traumatic encephalopathy. Recent research suggests that a combination of pharmacotherapy and cognitive therapy may effectively reduce symptoms and improve the quality of life in individuals with chronic traumatic encephalopathy.

\section{INTRODUCTION}

Chronic traumatic encephalopathy (CTE) is a neurodegenerative disease that is commonly observed in professional athletes, military veterans, and other individuals who have been subjected to repetitive brain injuries. Approximately 42 million people worldwide suffer from brain injury every year, which increases their risk of developing chronic traumatic encephalopathy later in life (Gardner \& Yaffe, 2015).

The main symptoms associated with the disease are profound memory loss, motor deterioration, unexplained aggression, depression, and suicidality. These cognitive and behavioral symptoms are also accompanied by biological changes in the brain. Similar to Alzheimer's disease, CTE is primarily characterized by an accumulation of tangles of protein, although the distribution of these tangles throughout the brain is unique to each dis-

\footnotetext{
${ }^{1}$ Department of Neuroscience, Carleton University, 1125 Colonel By Drive, Ottawa, ON K1S 5B6

*To whom correspondence should be addressed: christina.milani@carleton.ca
}

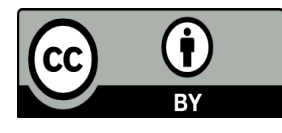

Except where otherwise noted, this work is licensed under https://creativecommons.org/licenses/by/4.0/

doi:10.22186/jyi.33.4.83-86 ease (Walker \& Tesco, 2013).

Significant attention was directed towards chronic traumatic encephalopathy when Dr. Bennet Omalu discovered the disease in a brain autopsy of former National Football League athlete Mike Webster, whose cognitive abilities had drastically declined following his retirement. Numerous indicators of significant brain deterioration were observed in Webster's autopsy, which was suggested to be accountable for his cognitive dysfunction in his later years (Omalu et al., 2005). Since this initial autopsy, $96 \%$ of professional athletes who have been examined for CTE by autopsy have been tested positive for the disease. Although CTE appears to be most prevalent among American football athletes, it is not restricted to this group of individuals. It is suggested that any individual who has been subjected to extensive brain injury throughout their life, including victims of abuse, can develop CTE (Baugh et al., 2012).

Although many great strides have been made in the progression of research on CTE, there is still much that remains unclear about the disease. Currently, there is no formal diagnosis that can be made while the individual is still alive. A post-mortem diagnosis can be performed by an autopsy, which allows for the identification of neuropathological markers of the disease. These markers include the presence of TAR DNA-binding protein 43 (TDP-43), a diffuse spread of hyperphosphorylated tau protein, and enlarged ventricles (Gavett, Stern, \& McKee, 2011). Further- 
more, there are no established treatment or rehabilitation protocols for individuals who are suspected to have the disease. The goal of current research on chronic traumatic encephalopathy is to investigate prospective solutions to these gaps in knowledge, and this review will discuss recent findings in this area.

\section{Diagnosing Chronic Traumatic Encephalopathy}

Although there have been numerous suggested guidelines for diagnosing CTE in vivo based on neuropsychological observations and life history, there are currently no widely accepted pre-mortem diagnostic criteria for the disease. The emergence of more sophisticated technology is creating opportunities for advancing in vivo diagnostic methods, particularly with regards to advancements in neuroimaging techniques. These advancements enable more detailed evaluations of the features of CTE, which are greatly beneficial for further understanding the neuropathological correlation of the disease and how they are unique from other neurodegenerative diseases.

Traditional structural neuroimaging techniques such as computed tomography (CT) and MRI that are often used in clinical assessments to evaluate gross anatomical changes are unable to effectively detect many of the pathological features of the disease that can only be observed at the cellular level, such as the aforementioned TDP-43 and hyperphosphorylated tau protein. However, DTI has become a popular tool for assessing brain injury as it is specialized for detecting abnormalities in brain white matter, which are common in cases of CTE as a result of the mechanical stress on axons following injury (Sundman, Doraiswamy, \& Morey, 2015). Assessing white matter integrity can provide insight into the severity of brain damage, and may be a useful tool in identifying individuals at risk of developing CTE.

In addition to DTI, functional neuroimaging techniques have demonstrated great promise in establishing connections between the neuropathology and symptomatology of CTE. Functional neuroimaging uses various techniques to assess brain activity, typically during the performance of a specific task. Positron emission tomography, or a PET scan, uses radioisotopes to measure the amount of glucose being taken up by regions of the brain, which is indicative of activity level. Barrio et al. (2015) used PET scans to investigate the differences in brain activity between retired football players who exhibited symptomatology associated with CTE, confirmedly diagnosed Alzheimer's patients, and normal controls. The study employed the radioisotope [F-18]FDDNP because it has a high affinity for insoluble protein aggregates, which are trademark features of both CTE and Alzheimer's disease. The neuroimaging results revealed significantly different signaling patterns across all three conditions. In the group of retired football players suspected to be tested positive for CTE, tau protein aggregates were observed to congregate in subcortical areas and limbic structures, namely in the amygdala. In contrast, the results of the Alzheimer's group indicated tau protein aggregates predominantly in medial temporal regions, with minimal involvement of subcortical structures. These findings are highly valuable as they not only demonstrate that neuroimaging tech- niques can potentially be used in the detection of chronic traumatic encephalopathy in vivo, but also to distinguish the neuropathology of CTE from other neurodegenerative disorders.

Another functional neuroimaging technique that has shown promise in identifying features of CTE is fMRI, which measures the levels of oxygen concentration in regions of the brain in response to a certain task or external stimuli. Brain regions with increased oxygen concentrations are suggested to respond with increased activity in comparison to regions with lower oxygen concentrations. Ford, Giovanello, and Guskiewicz's experiment (2013) was the first to use fMRI to assess differences in brain activity during memory tasks in football players who had been subjected to multiple concussions. The study compared two groups: football players who had experienced more than three concussions during their careers in sports and football players who had experienced less than three concussions. The results indicated no significant differences in performance on memory tasks between the two groups, although notable differences in neural activity were observed in the fMRI results. The low-frequency concussion group displayed more neural activity during relational memory tasks in the parahippocampal gyrus and the inferior parietal cortex. Previous research has suggested that these regions are associated with relational memory tasks in typical individuals. In contrast, the high-frequency concussion group recruited more neural activity from regions of the prefrontal cortex for the same relational memory tasks. The authors hypothesized that this may be due to the fact that the brain regions that are typically active during relational memory tasks were damaged in the highfrequency concussion group, thus explaining the lack of activity in these regions. Further investigation into the long-term effects of this differential neural activity during memory tasks is warranted, as this study did not observe any significant differences in functionality. These findings suggest that the restructuring of neural connections are likely to occur following repetitive brain injury, and support the hypothesis that there are discrepancies in the neurobiology of repetitive versus acute brain injury. This may have implications for the in vivo imaging of neural functioning in individuals who have been subjected to repetitive brain injury and who are suspected to test positive for CTE.

\section{Prospective Treatment Methods for Chronic Traumatic En- cephalopathy}

Just as there is a lack of pre-mortem diagnostic criteria for CTE, the same is true for treatment methods. The prospective treatment methods for CTE are predominantly preventative in nature by aiming to target and alleviate the adverse neurobiological outcomes of brain injury before they can become pathological and manifest as neurodegeneration. The investigation of pharmacological agents as candidates for the treatment of concussive brain injury has been a popular area of research, and there are several studies that have begun to investigate the use of pharmacological agents to directly target the neuropathology of chronic traumatic encephalopathy itself, rather than the preliminary features of brain injury. 
Zhang and colleagues observed that pharmacologically inhibiting the enzyme monoacylglycerol lipase, which plays an important role in degrading endocannabinoid neurotransmitters, significantly reduced the release of pro-inflammatory cytokines and suppressed the phosphorylation of tau protein in a mouse model with repetitive brain injury (Zhang, Teng, Song, Hu, \& Chen, 2015). Endocannabinoid neurotransmitters such as 2-arachidonoylglycerol are known to have anti-inflammatory properties, and these findings suggest implications of the endocannabinoid system in the management and reversal of CTE-like neuropathology. Additional research has demonstrated that administering the pharmacological agent JZL184, another monoacylglycerol lipase inhibitor, to rats which had undergone experimental mild traumatic brain injury significantly reduced neuroinflammation, glutamate excitotoxicity, and behavioural impairments associated with brain injury (Mayeux, Katz, Edwards, Middleton, \& Molina, 2017). These animal studies provide evidence for the neuroprotective role of the endocannabinoid system in cases of repetitive brain injury. Despite these promising findings, these types of drugs have yet to progress into human clinical trials as enhancing neurotransmission in the endocannabinoid system may have adverse effects including cognitive and sensorimotor impairments (Di Marzo, 2008).

Another experiment using a mouse model of traumatic brain injury revealed that administering lithium significantly reduced tau neuropathology in the thalamus and improved performance on spatial learning tasks (Yu, Zhang, \& Chuang, 2012). Although this study chiefly focused on the ability of lithium to reduce the presence of beta-amyloid plaques in the brain, a feature that is more consistent with Alzheimer's disease, the fact that an attenuation of tau neuropathology was also observed suggests that these findings may also be relevant to CTE. Additional research on using lithium as a treatment for brain injury and CTE is warranted as lithium is known to have psychotropic effects, including producing symptoms of dysphoria and cognitive slowing (Moncrieff, Cohen, \& Porter, 2013). Considering that cognitive disturbances and depression, which are often accompanied by dysphoria, are symptoms of brain injury and CTE, these effects of lithium may pose obstacles for its usage as a treatment method. These experiments are notable as they indicate that neurodegenerative markers of CTE may have the capacity to be reversed pharmacologically.

\section{DISCUSSION}

There have been many relevant research findings concerning the underlying mechanisms of CTE and implications for diagnosis and treatment. Functional neuroimaging techniques have drastically altered the way that the components of the disease can be investigated, and research has demonstrated a potential role for certain pharmacological agents in the treatment of the disease. In particular, several researchers are focusing on agents that enhance the anti-inflammatory properties of the endocannabinoid system.

Several limitations exist in terms of investigating prospective treatment methods for CTE. For one, the lack of a standardized pre-mortem diagnostic criteria for the disease makes it difficult to formulate a suitable treatment. Furthermore, there are obstacles with translating the existing pharmacological treatment research to human populations due to the known side effects of the proposed drugs. The aforementioned findings are promising, however there is still much that is largely unknown. There are some established hypotheses that aim to explain how brain injury can predispose an individual to developing CTE, although the specific mechanisms by which this occurs remain unclear. Moreover, there is currently no pre-mortem diagnostic protocol for the disease, despite the fact that there is substantial evidence suggesting that in vivo diagnoses are possible. Future research on the relationship between repetitive brain injury and disease progression is warranted, in addition to further investigation into the formation of a standardized diagnostic criteria and effective treatment methods for the disease.

\section{REFERENCES}

Barrio, J. R., Small, G. W., Wong, K.-P., Huang, S.-C., Liu, J., Merrill, D. A., ... Kepe, V. (2015). In vivo characterization of chronic traumatic encephalopathy using [F-18] FDDNP PET brain imaging. Proceedings of the National Academy of Sciences of the United States of America, 112(16), E2039-47. doi:10.1073/pnas.1409952112

Baugh, C. M., Stamm, J. M., Riley, D. O., Gavett, B. E., Shenton, M. E., Lin, A., .. Stern, R. A. (2012). Chronic traumatic encephalopathy: neurodegeneration following repetitive concussive and subconcussive brain trauma. Brain Imaging and Behavior, 6(2), 244-254. doi:10.1007/s11682-012-9164-5

Di Marzo, V. (2008). Targeting the endocannabinoid system: to enhance or reduce? $\mathrm{Na}$ ture Reviews Drug Discovery, 7(5), 438-455. doi:10.1038/nrd2553

Ford, J. H., Giovanello, K. S., \& Guskiewicz, K. M. (2013). Episodic memory in former professional football players with a history of concussion: an event-related functional neuroimaging study. Journal of Neurotrauma, 30(20), 1683-701. doi:10.1089/neu.2012.2535

Gardner, R. C., \& Yaffe, K. (2015). Epidemiology of mild traumatic brain injury and neurodegenerative disease. Molecular and Cellular Neurosciences, 66, 75-80. doi:10.1016/j.mcn.2015.03.001

Gavett, B. E., Stern, R. A., \& McKee, A. C. (2011). Chronic traumatic encephalopathy: a potential late effect of sport-related concussive and subconcussive head trauma. Clinics in Sports Medicine, 30(1), 179-88. doi:10.1016/j.csm.2010.09.007

Mayeux, J., Katz, P., Edwards, S., Middleton, J. W., \& Molina, P. E. (2017). Inhibition of Endocannabinoid Degradation Improves Outcomes from Mild Traumatic Brain Injury: A Mechanistic Role for Synaptic Hyperexcitability. Journal of Neurotrau$m a, 34(2)$, 436-443. doi:10.1089/neu.2016.4452

Moncrieff, J., Cohen, D., \& Porter, S. (2013). The psychoactive effects of psychiatric medication: the elephant in the room. Journal of Psychoactive Drugs, 45(5), 409-15. doi:10.1080/02791072.2013.845328

Omalu, B. I., DeKosky, S. T., Minster, R. L., Kamboh, M. I., Hamilton, R. L., \& Wecht, C. H. (2005). Chronic traumatic encephalopathy in a National Football League player. Neurosurgery, 57(1), 128-34-34. Retrieved from http://www.ncbi.nlm.nih. gov/pubmed/15987548

Sundman, M., Doraiswamy, P. M., \& Morey, R. A. (2015). Neuroimaging assessment of early and late neurobiological sequelae of traumatic brain injury: implications for CTE. Frontiers in Neuroscience, 9, 334. doi:10.3389/fnins.2015.00334

Walker, K. R., \& Tesco, G. (2013). Molecular mechanisms of cognitive dysfunction following traumatic brain injury. Frontiers in Aging Neuroscience, 5, 29 doi:10.3389/fnagi.2013.00029

Yu, F., Zhang, Y., \& Chuang, D.-M. (2012). Lithium reduces BACE1 overexpression, $\beta$ amyloid accumulation, and spatial learning deficits in mice with traumatic brain injury. Journal of Neurotrauma, 29(13), 2342-51. doi:10.1089/neu.2012.2449

Zhang, J., Teng, Z., Song, Y., Hu, M., \& Chen, C. (2015). Inhibition of monoacylglycerol lipase prevents chronic traumatic encephalopathy-like neuropathology in a mouse model of repetitive mild closed head injury. Journal of Cerebral Blood Flow and Metabolism, 35(3), 443-53. doi:10.1038/jcbfm.2014.216 